income, education, duration of symptoms before seeking medical treatment, duration of symptoms before the introduction of DMARDs, and specific DMARDs used.

\section{OP0145 GENE-ENVIRONMENT AND DISEASE INTERACTIONS: IS IT SMART TO USE COHORT STUDIES?}

TWJ Huizinga, M Kloppenburg. Department of Rheumatology and Epidemiology, Leiden University Medical Center, Leiden, The Netherlands

10.1136/annrheumdis-2001.78

Although much progress has been made in understanding the pathophysiology of RA, very few data are present on the cause of the disease. During the last years genetic factors (HLA, the X -chromosome) and environmental factors (smoking) have been identified as factors that are more often present in RA patients than in controls. By using a cohort study that enrols all patients with arthritis of less than two years of disease duration (The Leiden Early Arthritis Clinic), we were able to identify additional risk factors to develop RA such as a reduced fecundity in RA patients. However the current methodology to identify risk factors can be questioned. This presentation will focus on the alternative techniques such as case-case series or case-control studies as a more efficient approach to identify risk factors to develop RA.

\section{Infectious trigger mechanisms in R. D. - Thursday June 14, 10.00-11.45/Forum Hall}

\section{SP0015 THE DETECTION OF MICROBIAL DNA IN THE JOINT}

J Gaston. Rheumatology, University of Cambridge, Cambridge, UK

10.1136/annrheumdis-2001.79

Lyme disease, Whipple's disease and reactive arthritis are clear examples of forms of arthritis caused by bacterial infection, but the idea that infection might be involved in diseases like rheumatoid arthritis (RA) has a long history. Although joints in all of these conditions are usually "sterile" (i.e. no bacteria can be cultured from the joint), molecular methods of detection have demonstrated the presence of bacterial nucleic acids. The fact that ribosomal RNA (rRNA) genes from all bacteria have highly conserved sequences interspersed with species-specific sequences makes it possible to use polymerase chain reaction (PCR) techniques to detect any bacteria present in synovium and synovial fluid by amplification using "universal" primers which identify the conserved sequences, and then sequencing the products to determine the bacteria from which they arises. When this technique has been applied to synovium from RA patients, multiple species of bacteria have been identified, both by PCR (showing the presence of bacterial DNA) and RT-PCR; the latter implies the presence of live bacteria since bacterial rRNA is unstable, unlike DNA. These results cannot be accounted for by artefactual contamination, and suggest that resident bacteria such as gut and skin-derived organisms can find their way to the joint, most probably within phagocytes which have previously engulfed them and then been recruited to the inflamed joint. The same results were obtained when synovial fluid from reactive arthritis patients was tested. However in reactive arthritis, by using species-specific primers, it was also possible to detect the arthritistriggering organism (Chlamydia, Yersinia) in a proportion of cases. The specific organism was not detected simply by sequencing the product obtained using universal primers, which suggests that the specific organism is a minor component of the total bacterial colonisation of the joint.

Bacterial products such as DNA, LPS and protein antigens may contribute to joint inflammation both as targets of specific immune responses and as adjuvants. Nevertheless, reactive arthritis and RA differ in that the former usually resolves spontaneously; thus the presence of bacteria is not sufficient to cause chronic inflammation and other mechanisms which engender chronicity must be important in RA. Against a background of commensal bacteria it may be difficult to detect specific diseaseassociated organisms in RA, since specific organisms are in a minority in the joint even in diseases such as reactive arthritis in which they are clearly important in pathogenesis.

\section{SP0016 THE SPONDYLOARTHROPATHIES AS A PARADIGM FOR THE INDUCTION OF AN AUTOIMMUNE RESPONSE BY BACTERIA}

J Sieper. Rheumatology, University Hospital Benjamin Franklin and German Rheumatology Research Center, Berlin, Germany

10.1136/annrheumdis-2001.80

The spondyloarthropathies (SpA) comprise ankylosing spondylitis (AS), reactive arthritis (ReA) or Reiter's syndrome, arthritis/ spondylitis with inflammatory bowel disease (IBD) and arthritis/ spondylitis with psoriasis. The main link between each other is the association with HLA-B27, the same pattern of peripheral joint involvement with an asymmetrical arthritis predominantly of the lower limbs, and the possible occurrence of sacroiliitis, spondylitis, enthesitis and uveitis. Bacteria play a central role in the pathogenesis of the SpA. This is most obvious in ReA which is triggered by preceding bacterial infections of the urogenital or gastroenteral tract. In arthritis/spondylitis associated with IBD a causative role of gut bacteria is also assumed which can stimulate the cellular immune sytem because the breakage of the gut barrier. Because between $20-50 \%$ of HLA-B27-positive patients with ReA or IBD move on to AS a causative or triggering role of bacteria can also be assumed in AS. Due to more recent studies using new imaging techniques such as MRI and immunohistological investigations it can now be assumed that the immunopathology of SpA occurs typically at the interphase of bone and cartilage and that synovitis is only a secondary event. Although at the moment it cannot be excluded that bacteria or pieces of bacteria persist in these structures this seems to be unlikely. Thus, an autoimmune response triggered by a bacterial infection seems to be more likely. Cartilage derived proteins such as aggrecan could be, according to studies in animal models of SpA and to studies in SpA patients, a primary target of this autoimmune response. Such a response could be due to cross-reactivity with a bacterial antigen. This possible course of events leading to a chronic immune response in SpA has to be proven in the near future. The identification of target antigens would offer the possibility to switch off the antigen-specific immune response as a new exciting treatment option. 